\title{
Article \\ Effect of Fuel Composition on Carbon Black Formation Pathways
}

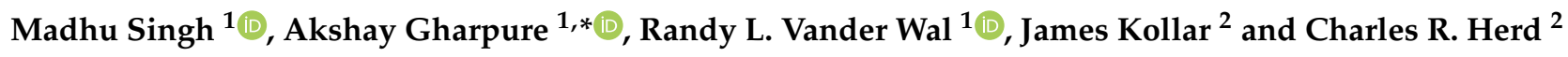 \\ 1 The John and Willie Leone Family Department of Energy and Mineral Engineering and the EMS Energy \\ Institute, Pennsylvania State University, University Park, PA 16802, USA; \\ madhusingh@alumni.psu.edu (M.S.); ruv12@psu.edu (R.L.V.W.) \\ 2 Birla Carbon, 1800 West Oak Commons Court, Marietta, GA 30062, USA; james.kollar@adityabirla.com (J.K.); \\ charles.herd@adityabirla.com (C.R.H.) \\ * Correspondence: apg86@psu.edu
}

check for updates

Citation: Singh, M.; Gharpure, A.; Vander Wal, R.L.; Kollar, J.; Herd, C.R. Effect of Fuel Composition on Carbon Black Formation Pathways. Appl. Sci. 2022, 12, 2569. https:// doi.org/10.3390/app12052569

Academic Editors: Patrizia Minutolo and Mario Commodo

Received: 3 February 2022

Accepted: 24 February 2022

Published: 1 March 2022

Publisher's Note: MDPI stays neutral with regard to jurisdictional claims in published maps and institutional affiliations.

Copyright: (C) 2022 by the authors. Licensee MDPI, Basel, Switzerland. This article is an open access article distributed under the terms and conditions of the Creative Commons Attribution (CC BY) license (https:// creativecommons.org/licenses/by/ $4.0 /)$.

\begin{abstract}
Differences in lamellae length, stacking, and particularly a difference in the core-to-shell radial structure are observed for carbon blacks produced using different feedstocks. Carbon black (CB) produced using a coal tar (CT) feedstock formed particles with amorphous cores exhibiting a sharp transition to extended lamellae oriented about the periphery of the particle. In contrast, the carbon black produced from fluidized catalytic cracker (FCC) decant oil as feedstock formed particles with a single nucleated core possess a rather uniform radial transition-reflecting the presence of ordered, concentric lamellae across most of the particle radius. Minimal disorder was observed in the core while the undulations in perimeter lamellae were fewer. Our interpretation for these structural dissimilarities is premised on differences in fuel composition, specifically component classes as found by saturate, aromatic, resin, asphaltene (SARA) analysis. These in turn lead to variation in the relative rates of particle nucleation and particle growth by pyrolysis products, moderated by temperature. Electron energy loss spectroscopy reveals radial variation in the $\mathrm{sp}^{2}$ content between the different feedstocks consistent with observed nanostructures. Collectively these results are interpreted in terms of an offset in nucleation and growth-dependent upon the relative contributions of feedstock aromatic content and pyrolysis processes to particle nucleation and growth. To further test the postulate of different formation conditions for the two carbon blacks pulsed laser annealing was applied. The high temperature heating accentuated the dissimilarities in nanostructure and chemistry-leading to stark dissimilarities. These differences were also manifested by comparing oxidative reactivity.
\end{abstract}

Keywords: carbon black; nanostructure; HRTEM; laser annealing; flame formed carbon; fuel composition

\section{Introduction}

Reactive dimerization by the formation of covalent-covalent bonds is becoming recognized as a viable pathway and likely a dominant route to nuclei formation [1,2]. Notably, this pathway does not require exceptionally large Polycyclic Aromatic Hydrocarbons (PAHs) to realize sufficient van der Waals forces at flame temperatures [3-6]. Underscoring this new path are the observations that soot particles appear sooner than even moderately sized PAHs (e.g., pyrene) along the centerline of a diffusion flame [2]. Additionally, atomistic simulations provide evidence for $\mathrm{PAH}$ growth involving radicals that bypass the traditional small molecule growth pathways [3]. Most importantly, this mechanism implicates fuel-sourced PAHs as a significant source of soot nuclei. Small PAHs prevalent in the fuel provide high initial concentrations to feed nuclei formation, thereby affecting early-stage particle growth dynamics. In parallel, the recent study by Kholghy et al. showed that soot nucleation must involve strong chemical bond formation between dimers [4]. Initially, unstable dimers are formed through reactive bonding — covalent bonds are formed 
through a sequence of hydrogen abstraction and carbon-carbon bond formation, or dehydrogenation, which stabilizes the dimers. The evolution of soot concentration is predicted through comparison to measurements. Nucleation involving all PAHs rather than a single one has been found essential for predicting PAH and soot concentrations.

Alternatively, very large PAHs can contribute to particle nucleation by van der Waals forces, but these are generally in low concentrations-requiring first their formation in the flame environment or reactor. Similarly, for feedstocks with lower aromatic concentrations (and correspondingly higher paraffinic and naphthenic content) - pyrolysis reactions followed by aromatic ring building reactions are needed to raise aromatic concentrations to sufficient levels for reactive condensation to be an effective path to nuclei formation [7,8]. These degradative and growth reaction sequences impose a kinetic delay-governed by temperature [9].

In this study, two types of carbon blacks are compared for their physical and chemical attributes, including morphology, nanostructure, and composition. These carbon blacks are formed under similar reactor conditions, with feedstock composition being the only significant variable. One carbon black is formed using coal tar as the feedstock (referred to as CT-CB), while the other carbon black is formed using fluid catalytic cracking decant oil (referred to as FCC-CB). It is difficult to ascribe a specific aspect of nanostructure to a particular component class in the original fuel given several component classes and their chemical and physical transformations by concerted pyrolysis and growth reactions. Still, recognized differences between classes of compounds present within CT versus FCC feeds may now be understood in the context of newly recognized soot nucleation/formation pathways. Specifically, these advanced pathways begin with aromatics and the resonance stabilized radical reactions between them and other small hydrocarbons $[5,6]$. Such low energy pathways negate the preconditions for high temperature, high $\mathrm{H}$-atom concentrations in order to form nuclei [7] while also removing the energetic and kinetic restriction of only large PAHs serving as soot nuclei [8].

The two feedstocks lead to significant differences in primary particle nanostructure and $\mathrm{sp}^{2} / \mathrm{sp}^{3}$ chemistry—as observed and characterized by electron-based microscopic and spectroscopic measurements. The influence of the source fuel extends to oxidative reactivity, demonstrated here by isothermal thermogravimetric analysis conducted at $\sim 500{ }^{\circ} \mathrm{C}$. To further differentiate nanostructure and chemistry differences, pulsed laser annealing is applied. Pulsed laser annealing has been recently shown to derivatize combustion generated carbons [9]. Here it is applied to enhance nanostructure and chemical differences between two carbon blacks thereby aiding structural differentiation by feedstock.

\section{Materials and Experimental Methods}

\subsection{Feedstocks}

The two carbon blacks used in this study are derived from different feedstocks, described briefly as follows.

CT-CB is derived from a coal-tar based feed. Coal tar is an extremely complex mixture, containing 4, 5, 6, and even 7-ring aromatics, as well as methylated and poly-methylated derivatives with SARA analysis differentiating saturates and aromatic. Other components can include heterocyclic compounds such as pyridine, benzene-toluene-xylene (BTX) compounds, cumenes, and naphthalenes [10].

FCC-CB is derived from fluid catalytic cracking of decant oil. SARA analysis results highlight the key differences relative to the CT material as higher saturate and lower aromatic content. FCC decant oils can contain an aromatic distribution ranging from single to 4-ring compounds, potentially including some naphthenic rings. Reflecting catalytic origins, the lighter aromatic fraction may bear varied alkyl groups as reflected by the SARA analysis [11].

Table 1 summarizes the results of the analysis for saturates, aromatics, resin, and asphaltene content (SARA) for these feedstocks. The sulfur content for the CT was $0.609 \mathrm{wt} \%$ and $3.15 \mathrm{wt} . \%$ for the FCC material. 
Table 1. Resolved compositions by SARA analysis.

\begin{tabular}{ccccc}
\hline Feedstock & Saturates & Aromatics & Resins & Asphaltenes \\
\hline Coal tar & 0.8 & 97.19 & 0.91 & 0.38 \\
FCC decant oil & 7.3 & 90.8 & 0.4 & 1.5 \\
\hline
\end{tabular}

These carbon blacks, formed with different fuel sources are compared using the following analytical techniques for their morphology and nanostructure.

\subsection{Transmission Electron Microscopy (TEM)}

TEM is done using an FEI Talos F200X equipped with a $200 \mathrm{keV}$ FEG source and a resolution of $0.12 \AA$. Samples were dispersed and sonicated in methanol before being dropped onto 300 mesh $\mathrm{C} / \mathrm{Cu}$ lacey TEM grids.

\subsection{Electron Energy Loss Spectroscopy (EELS)}

EELS was used to probe the bonding chemistry in the samples. The relative proportion of transitions where core electrons are promoted to unoccupied $\pi^{*}$ versus $\sigma^{*}$ states is a measure of $\mathrm{sp}^{2}$ and $\mathrm{sp}^{3}$ density of state. The energy loss from incident electrons as they travel through carbon typically occurs in the $284-305 \mathrm{eV}$ range. The transition in the $284-289 \mathrm{eV}$ region is attributed to $1 \mathrm{~s}$ to $\pi^{*}$ while $1 \mathrm{~s}$ to $\sigma^{*}$ transition occurs in the $290-305 \mathrm{eV}$ range. The energy loss measurements were taken using FEI Titan $\mathrm{G}^{2}$ microscope operated at $300 \mathrm{kV}$ in scanning transmission electron microscopy (STEM) mode for high spatial resolution. The beam was condensed in the region of interest such as core vs the edge of the particle to study the radial variation in the bonding chemistry. The instrument is equipped with a Gatan GIF Quantum electron filter, and the monochromator provides energy resolution close to $0.1 \mathrm{eV}$ with monochromator activated. A Shirley background was subtracted in the $284-305 \mathrm{eV}$ region, and a gaussian peak was fitted in each transition range. The peak intensity and full width at half maximum (FWHM) were allowed to vary, and the fit with the lowest residual value was selected for quantification. The $\mathrm{sp}^{2}$ and $\mathrm{sp}^{3}$ contents were estimated using the integrated area under the two transition peaks.

\subsection{Fringe Analysis}

The graphitic nanostructure can be characterized by its in-plane layer dimensions $\left(\mathrm{L}_{\mathrm{a}}, \mathrm{L}_{\mathrm{b}}\right)$, stacking height $\left(\mathrm{L}_{\mathrm{c}}\right)$, inter-planar spacing $(\mathrm{d})$, and tortuosity or curvature of the lamellae [12,13]. Lattice fringe length is the end-to-end distance measuring the fringe along its length. Longer lengths correspond to a higher degree of organization, and hence the material is considered more graphitic. Fringe tortuosity is defined as the ratio of the actual fringe length to the shortest distance between the endpoints of a carbon segment and provides a measure of the undulation of the layer planes.

\subsection{Thermogravimetric Analysis}

A TA instruments Thermogravimetric Analyzer (TGA) with simultaneous Differential Scanning Calorimetry (DSC) TGA-SDT Q600 is used as a bulk material characterization technique to study the response of the material to isothermal treatment at $500{ }^{\circ} \mathrm{C}$. The carbon blacks were heated at a ramp rate of $5{ }^{\circ} \mathrm{C} / \mathrm{min}$ until a $50 \%$ weight loss was attained.

\subsection{Laser Annealing}

The two carbon blacks are laser heated using 1 pulse of $170 \mathrm{~mJ} / \mathrm{cm}^{2}$ of the Nd:YAG laser, operating at a wavelength of $1064 \mathrm{~nm}$. The pulse duration is fixed at $8 \mathrm{~ns}$. Thus, the sample heats up rapidly in $<8 \mathrm{~ns}$ to temperatures $>3000{ }^{\circ} \mathrm{C}$ in an inert, and cools to atmospheric temperature over the next few microseconds after the laser pulse. The sample is heated directly on lacey C/Cu TEM grids. 


\section{Results and Discussion}

3.1. Carbon Black Aggregate Morphology and Particle Structure

TEM is used to visualize aggregate morphology and particle structure for the two carbon blacks, shown by the micrographs in Figure 1.

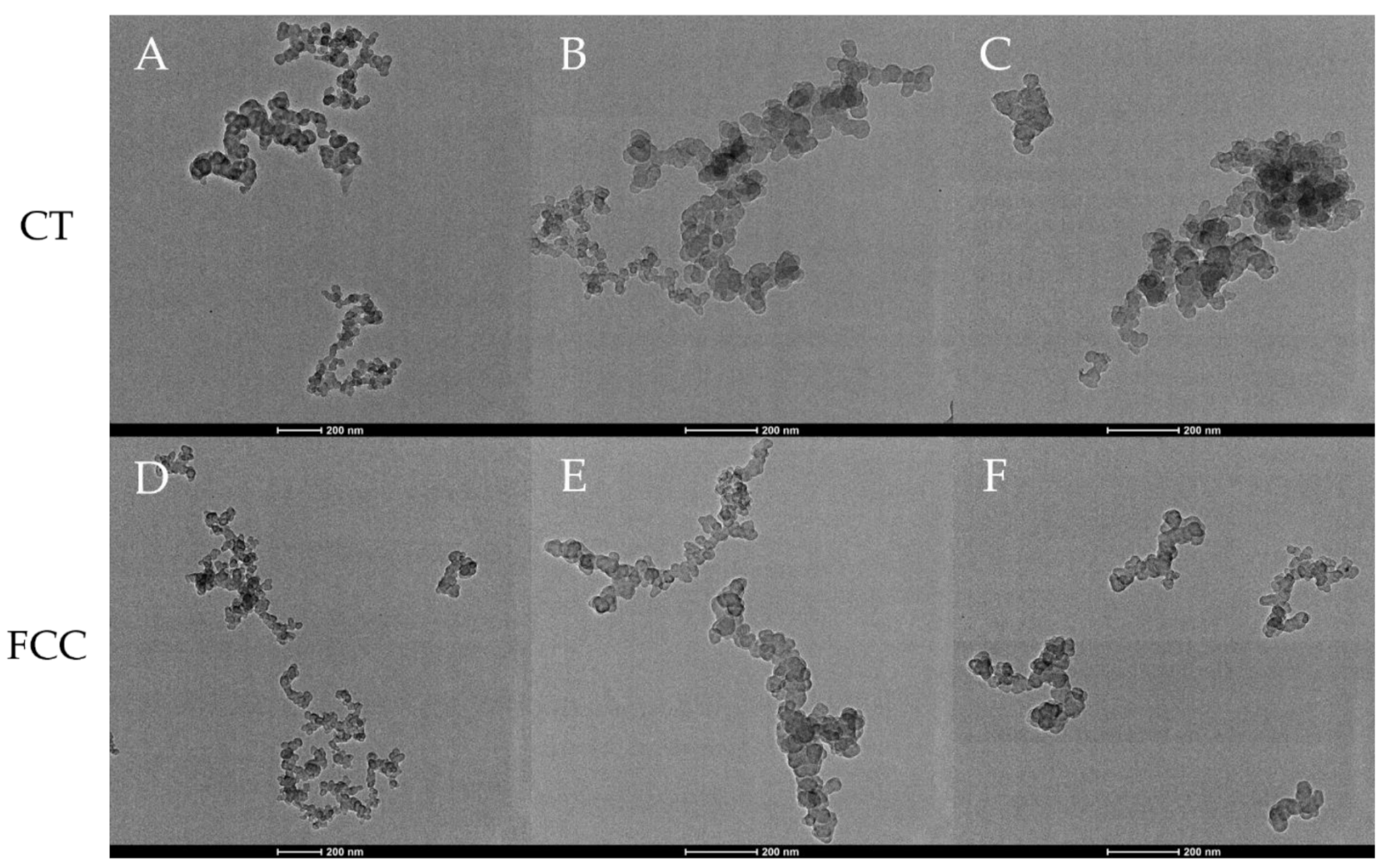

Figure 1. Aggregate morphology of (A-C) CT- CB and (D-F) FCC-CB.

Both image series show a range of aggregate sizes, consisting of a few to several dozen primary particles. Dendritic and compact morphologies are observed for aggregates from both feedstocks. While the degree of branching morphology appears to decrease with aggregate size, this is a consequence of the fewer primary particles within these aggregates but does not necessarily change the fractal descriptor. Gauging by mass thickness contrast, even large aggregates possess regions of compact primary clustering.

The primary particles are distinctly different between the two carbon blacks. While FCC-CB shows well-defined, pseudo-spherical primary particles typical of many commercial carbon blacks, CT-CB does not show such defined "particles". Figure 2 shows a visual comparison of structural differences between primaries for FCC- and CT-CB. 


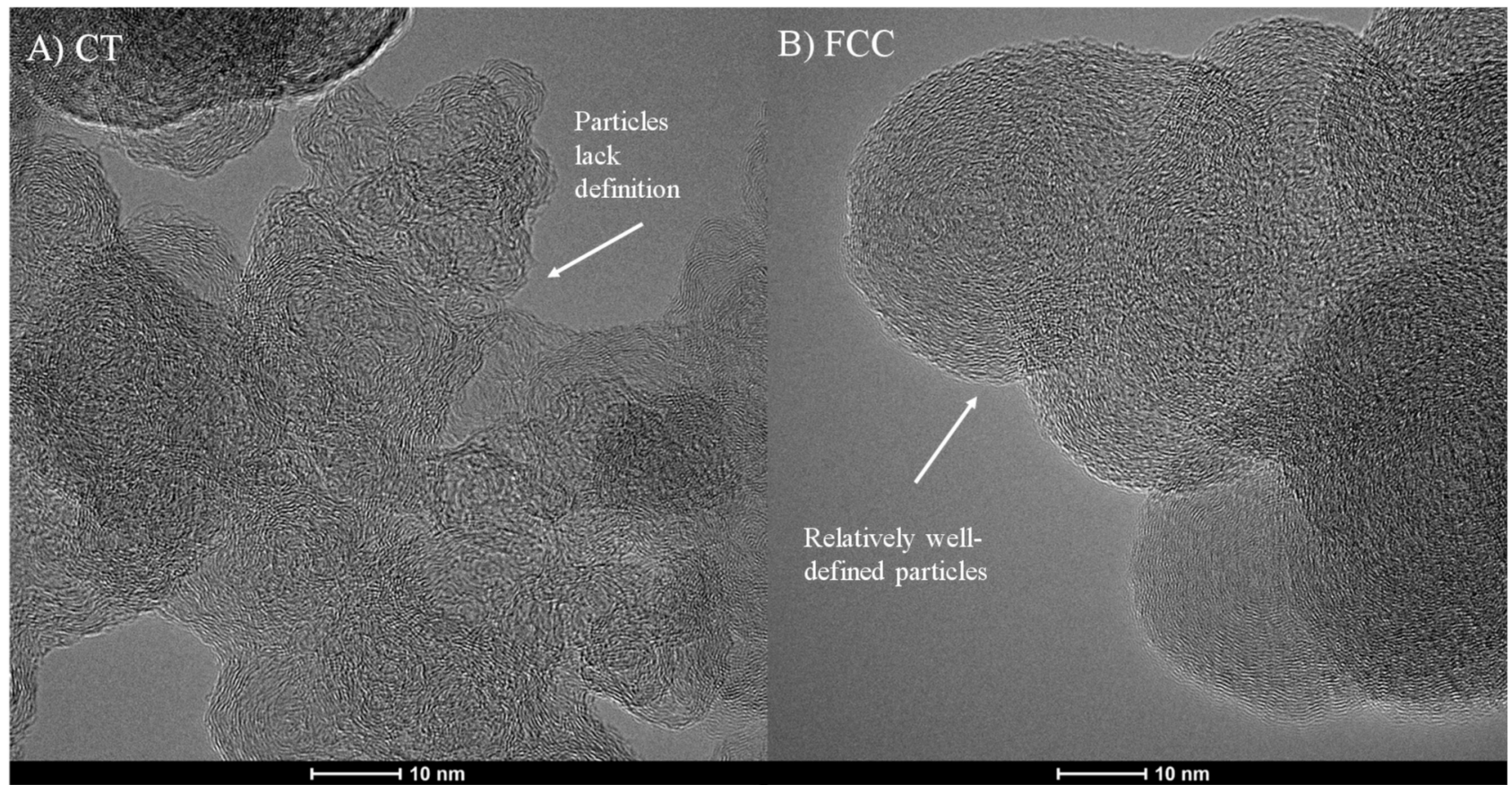

Figure 2. TEM micrographs showing particle structure for (A) CT- and (B) FCC-CB black.

\subsection{Carbon Black Nanostructure}

Carbon black nanostructure has previously been shown to be a function of its formation conditions $[14,15]$. Recently, we demonstrated the impact of different fuels on soot nanostructure [9].

Figure 3 shows high resolution transmission electron microscopy (HRTEM) micrographs of CT-CB versus FCC-CB. The CT-CB shows a disordered nanostructure within its particles. Lamellae orientation at the core of the particle is amorphous and unstructured. In contrast, FCC-CB particle cores lack visible disorder. Lamellae appear ordered, somewhat concentric, following the orientation of the perimeter lamellae that comprise its shell.

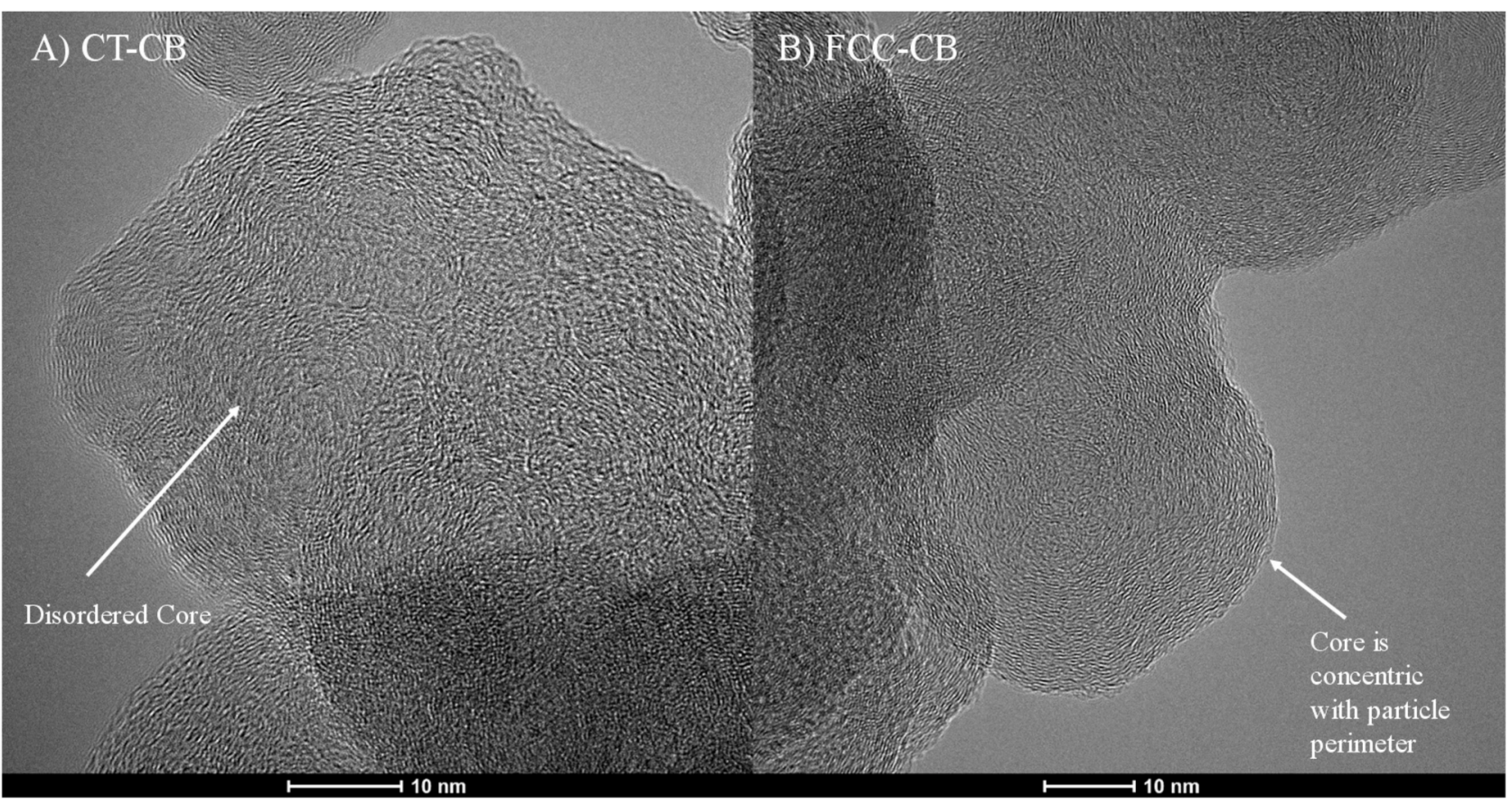

Figure 3. HRTEM micrographs showing nanostructure of (A) CT-CB and (B) FCC-CB. 
Carbon black nanostructure can be quantified by lattice fringe analysis for lamellae length and curvature. Fringe analyses for the two carbon blacks are compared next.

\subsection{Lattice Fringe Analysis}

Lattice fringes, formed by the constructive interference of the transmitted and diffracted beams in a TEM provide a reconstruction of the lamellae structure and can be used to quantify the nanostructure of flame formed carbons or carbon black and differentiate between materials based on their lamellae arrangement. For the CT- and FCC-CBs, lattice fringe analysis has further resolved, spatially, nanostructure differences between the particle's shell and core lamellae. Fringe length and tortuosity are quantified from HRTEM micrographs and compared in Figure 4.

CT-CB: Figure 4A shows differences in fringe lengths of the lamellae that comprise the particle's core and the particle's shell. Lamellae that comprise the particle's shell have longer (higher $\%>1 \mathrm{~nm}$ in length) fringes relative to the lamellae that make up its core as seen by TEM and indicated here by the positive \%'s for longer fringe lengths.

FCC-CB: Figure 4B shows a different plot of fringe lengths for FCC-CB's shell and core lamellae. One striking feature of this graph is the similarity between parameters for the lamellae that comprise the particle's shell and core, indicating radial uniformity across the particle.
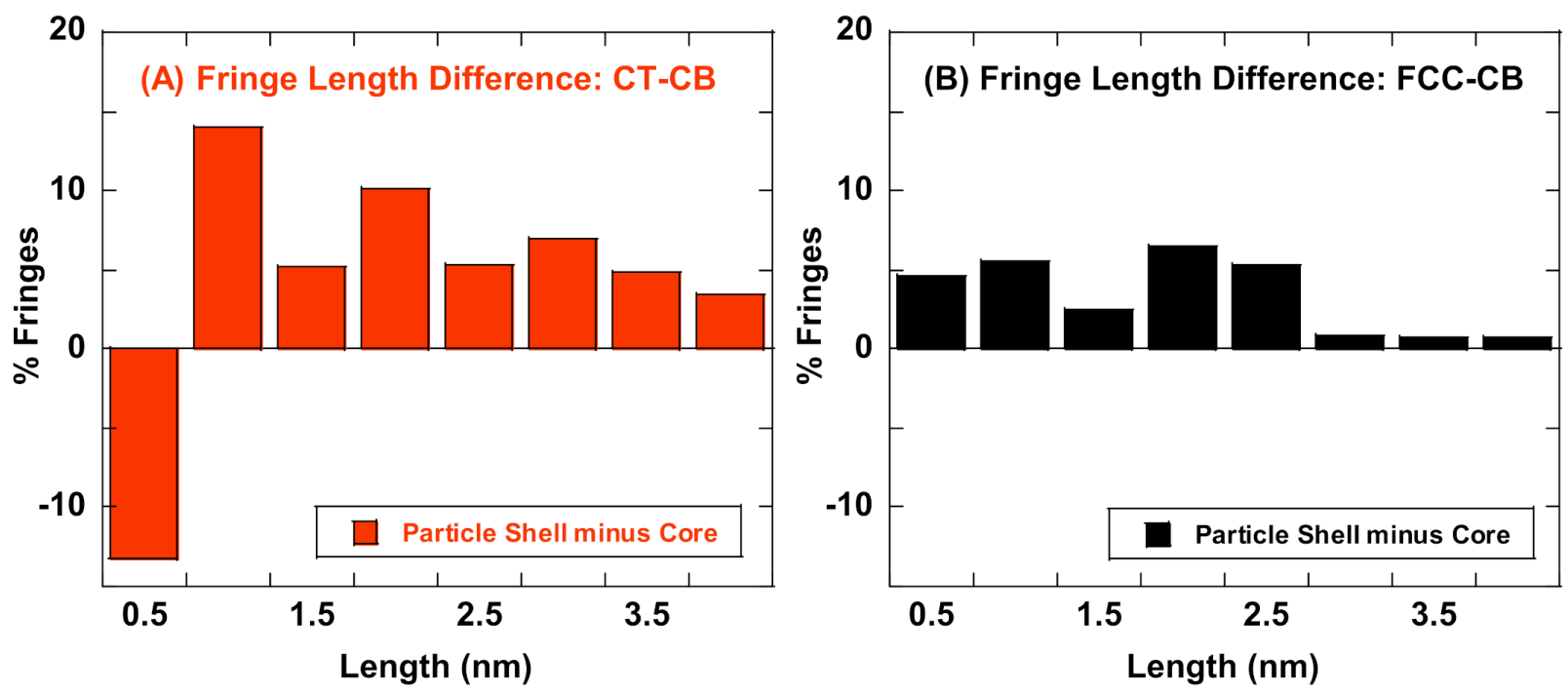

Figure 4. Lattice fringe analysis showing differences in fringe lengths of particle core lamellae subtracted from particle shell lamellae for (A) CT-CB and (B) FCC-CB blacks.

\subsection{Electron Energy Loss Spectroscopy (EELS)}

EELS is used to illustrate particle-to-particle variation (or lack thereof) of $\mathrm{sp}^{2}$ to $\mathrm{sp}^{3}$ hybridized carbon. Quantification in EELS is dependent on an appropriate calibration standard [16]. Graphitized carbon black has been used as the EELS calibration standard in this work with the assumption of $100 \% \mathrm{sp}^{2}$ content, its quasi-spherical geometry making it free of orientation effects-a potential disadvantage when using graphite as a standard [17].

For the carbon black samples in this work, EELS spectra were collected and averaged from 7-15 particles for each carbon. The integrated EELS intensities are the basis for differentiating $\mathrm{sp}^{2}$ and $\mathrm{sp}^{3}$ orbitals, based on the corresponding electronic transition intensities and associated energy loss of the incident electrons [16-18]. Normalized percentages are reported in Table 2. Commonly such values are reported after normalization by a graphitic standard to correct for aromatic ring (and hence orbital) orientations. As a common constant, it preserves the ordering but diminishes the differences in the unprocessed data, listed in Table 2, along with the corrected values normalized to the graphitic standard. The difference between particles originating from the varied feedstocks is apparent and 
consistent with projections for particle formation and growth based on the compositional differences.

Table 2. EELS analysis results for the three carbon blacks from the indicated feedstocks.

\begin{tabular}{ccc}
\hline & CT & FCC \\
\hline No. Measurements & 7 & 15 \\
Ratio: Edge/Center & 0.86 & 0.83 \\
Normalized Mean sp ${ }^{2}(\%)$ & $7.2 \pm 1.8$ & $24.4 \pm 1.6$ \\
sp $^{2}$ referenced value & 46 & 78 \\
Rel. Ratio & 1 & 3.4 \\
\hline
\end{tabular}

The CT-CB possesses the lowest $\mathrm{sp}^{2}$ content with higher $\mathrm{sp}^{3}$ bonding and hydrogen content. Differently, the FCC derived particles exhibit a substantially higher $\mathrm{sp}^{2}$ content. Interestingly the ET-CB particles exhibit an intermediate value of $\mathrm{sp}^{2}$ percent. Our inference is an intermediate compositional balance of aromatic and aliphatic components, but further resolution by feedstock analysis is not presently available.

CT- and FCC-CB have thus far displayed physical differences in primary particle size, nanostructure, and chemical bonding differences. Such divergence in structure and chemistry brought about by changing the feedstock can further influence material reactivity such as its response to thermal treatment. This is demonstrated in the subsequent section.

Thermal treatment is a widely used processing tool for carbon materials such as carbon black. Therein it is necessary to understand material response based on its initial chemistry and structure. Here CT- and FCC-carbon have been subjected to a moderate $\left(500{ }^{\circ} \mathrm{C}\right)$ oxidation under isothermal conditions using a TGA and a comparatively high-temperature $\left(>3000{ }^{\circ} \mathrm{C}\right.$ ) laser heat treatment. The response of the two carbon blacks is recorded and related back to their nanostructure and chemistry-demonstrating how reactivity is ultimately influenced by feedstock, mediated by particle nanostructure and chemistry.

\subsection{Carbon Black Formation-Dependence upon Fuel Compositional Differences}

CT composition is dependent upon both initial coal rank and pyrolysis conditions of temperature, rate, and quenching [18-20]. Yet general distinctions are remarkably consistent given the evolution of coals along the van Krevelen path and fit within the Seylar coal band [21]. Specifically, CT consists predominantly of small aromatics with ensuing pyrolysis reactions increasing their content facilitating their growth. Alkyl groups, ether linkages, etc. will have undergone cleavage during the destructive thermal pyrolysis [19,22]. Light volatiles and saturates arise by cleavage of such linkages and dealkylation reactions [23]. The significance of the high concentration of small aromatics and high level of methylation [24] is that these species will readily form resonance stabilized radicals [25,26]. Their low barrier to formation facilitates their participation in the resonance stabilized radical addition reactions considered central to soot nuclei formation and subsequent growth [6,27]. For such reactions, feedstock aromatics are a direct precursor. High temperatures activating sufficient $\mathrm{H}$-atom concentrations for hydrogen abstraction acetylene addition reactions (HACA mechanism) towards the buildup of the 1st aromatic ring and subsequent growth are not required [28]. It should also be noted that fractionation studies on CT have found a continuum of cuts ranging from naphtha, kerosene, diesel, heavy gas oil, and even a small resid fraction (a few wt.\%) [29]. Such a broad molecular size range is synergistic with the resonance stabilized radical formation path given aromatics in each cut.

The FCC feedstock also contains considerable aromatic content, but with saturate content in the form of long-chain paraffins, i.e., waxes and alkyl substituent groups on aromatics [24]. The paraffinic content lowers the overall aromatic concentration, slowing the rate of nuclei formation while requiring pyrolysis reactions to form aromatics thereby imposing a kinetic delay, $\tau$. Perhaps more importantly the saturate decomposition (whether as paraffins or substituent groups) generates $\mathrm{H}$-atoms and alkyl radicals that can cap aromatic radicals to restrict reactive radical addition reactions [30]. Preceding pyrolysis 
saturates can serve as H-donors to terminate aromatic radicals-restricting their clustering via radical chain reactions [31,32]. Though the reaction rates are fast and mechanistic steps few for these processes, any kinetic delay $(\tau)$ allows turbulent mixing with flame gases, temperature elevation, and homogenization of the reactive environment under which nuclei form and particle growth occurs [33].

Interpretation for the structural differences spanning nano- to micro-scales for the two carbon blacks is premised upon the feedstock compositional differences. Formation of carbon black using the two feeds is postulated to begin at different stages of the carbon black formation pathway for CT-CB and FCC-CB, illustrated by the schematic in Figure 5 -based on feedstock compositional differences as listed in Table 1, analogous to the well-known soot formation pathway [34].

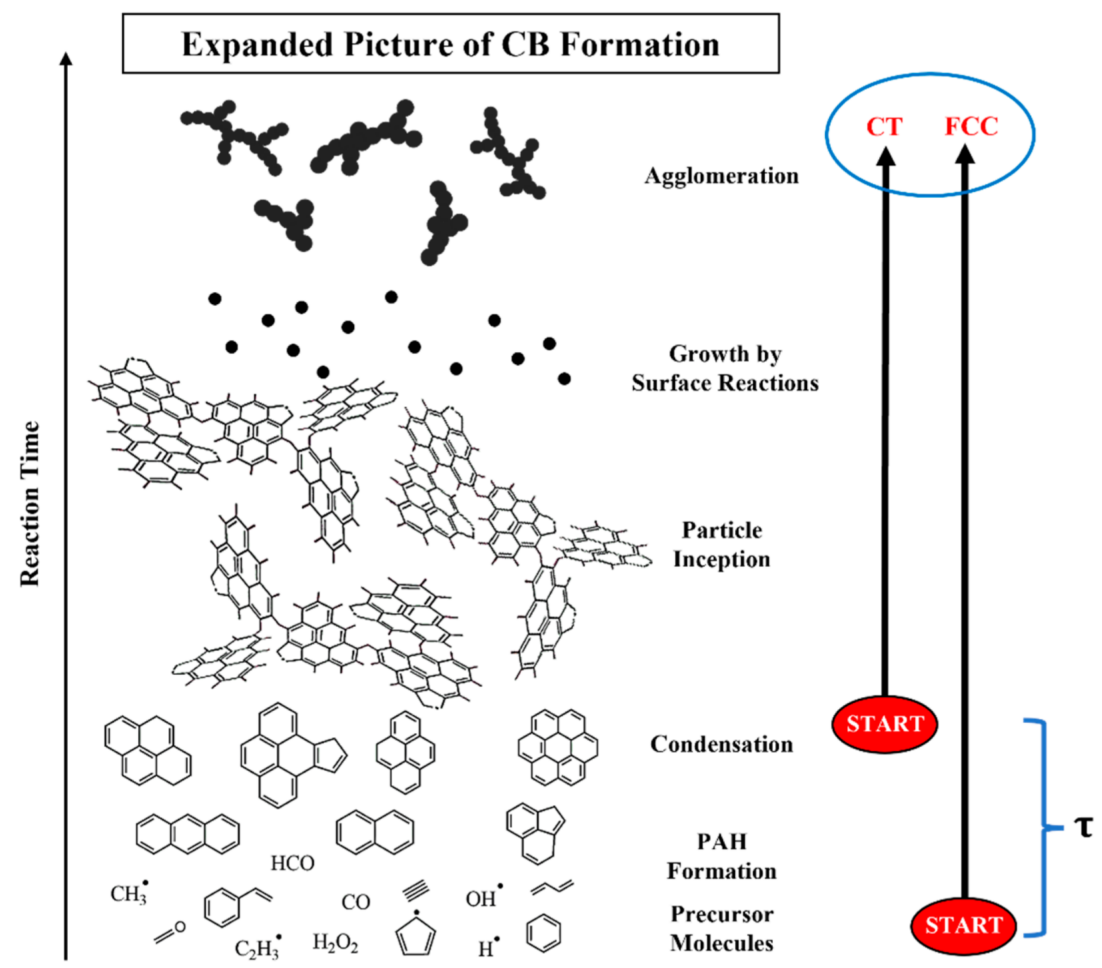

Figure 5. Carbon black formation pathway to illustrate the difference in the formation of CT- and FCC-CB.

\subsection{1. СТ-CB}

Coal tar is a high aromatic feedstock. As summarized earlier, aromatics can bypass the normal sequence of fuel pyrolysis, acetylene production, and benzene formation $[29,35]$. Forming resonance stabilized radicals at lower temperatures by reactions not restricted to $\mathrm{H}$-atom abstraction $[5,6]$, particle inception and growth can begin earlier and at lower temperatures than for a feedstock requiring such a reaction sequence [20,26]. With origin either from the feedstock or via a molecular breakdown/buildup reaction sequence, by a combination of physical and reactive condensation, aromatics readily form CB seed nuclei $[36,37]$. Further growth can immediately continue via coalescence of inception particles and by reactive aromatic condensation [5,6]. Rates for both processes are accelerated by the locally high concentration of seed nuclei and aromatics-as occurs when they originate from the feedstock. With inception beginning by rapid coalescence and occurring at lower temperatures, the particle core structure is poorly developed and will appear to have multiple nucleation centers-reflecting nuclei clustering - as shown in Figure 2, or disordered cores as in Figure 3. Additionally, feedstock aromatics will impart a higher $\mathrm{H} / \mathrm{C}$ content by alkyl and/or methylated substituents, these being partially preserved at the initially lower temperature-netting a higher $\mathrm{sp}^{3}$ content. With the rapid depletion of heavy aromatics, 
further $\mathrm{CB}$ growth occurs via fuel pyrolysis products. The induction time required for these pyrolysis reactions allows mixing with surrounding combustion gases, elevating the temperature field while diluting the growth species concentration. Surface lamellae growth occurs under a very different reaction environment: smaller growth species, e.g., acetylene, step-wise radical creation/addition mechanism, e.g., HACA, and higher temperature. These factors and particularly the latter favor development of extended lamellae with more order [28,38] — consistent with the observed radial variation in nanostructure to an ordered shell. The greater length and stacking of perimeter lamellae are indicators that the feed-derived growth species and temperature field are different for this stage of particle growth compared to earlier particle inception and initial growth. As inferred from the radial nanostructure of the $\mathrm{CB}$ particles, growth occurs under locally different chemical/temperature conditions. Different fuel components contribute to different stages of early versus latter particle growth - with aromatic content accelerating early inception and growth.

\subsubsection{FCC-CB}

FCC decant oil consists of higher paraffinic content such as branched compounds, alkylated aromatics, and waxes. The lower aromatic concentration delays inception by fuel-carried aromatic radical condensation. A contributing factor is radical capping by the saturate derived $\mathrm{H}$-atoms and alkyl radicals plus radical termination by $\mathrm{H}$-abstraction reactions from saturates. By this combination of physical dilution and radical termination reactions, particle inception and growth are delayed by the time required to reach sufficient aromatic concentrations for particle inception and growth. The kinetic time delay $(\tau)$ for pyrolysis reactions and subsequent aromatic buildup allows turbulence induced mixing to lower the feed concentration (by mixing with surrounding post-combustion gases) while simultaneously increasing the temperature. The net result is that smaller species (e.g., acetylene) contribute to early particle growth under higher temperature conditions than the aforementioned processes (early growth) of reactive coalescence and surface condensation of PAHs as for CT. More ordered lamellae form-beginning upon particle inception. A higher $\mathrm{sp}^{2} / \mathrm{sp}^{3}$ ratio results. More consistent chemical (aka growth species) and temperature environment produces primary particles with greater radial uniformity of nanostructure-as seen in Figures 2 and 3. Primary particles exhibit a single nucleus-as observed in Figures 2 and 3.

\subsection{Nanostructure Inferences Reconciled Using Oxidative Thermal Treatment on CT- and FCC-Carbon Black Nanostructure}

CT- and FCC-CB were treated isothermally at $500{ }^{\circ} \mathrm{C}$ in a TGA until a $50 \%$ weight loss was observed. Trends of weight loss and its derivative curve smoothed with respect to time are shown in Figure 6.
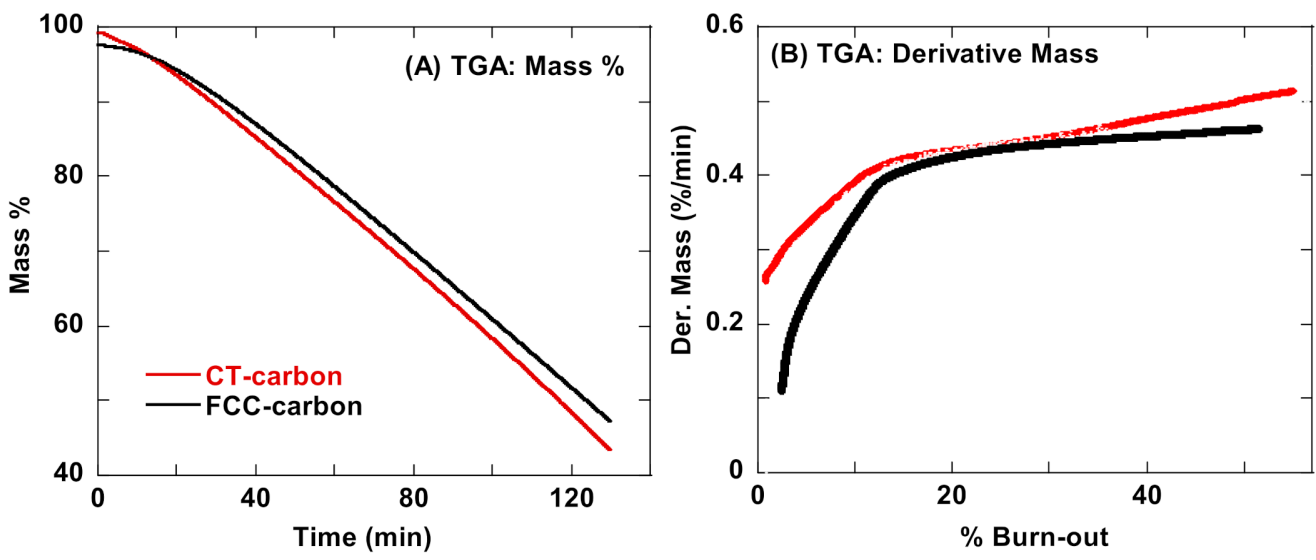

Figure 6. TGA (A) weight and (B) derivative weight analysis for CT- and FCC-CB. 
TGA weight loss under isothermal $\left(500^{\circ} \mathrm{C}\right)$ conditions in air shows the CT-CB to lose more mass relative to the FCC-CB. This is better illustrated in their derivative plots, where the percent mass lost by CT-CB increases with heat treatment time and percent burn-out. This agrees with the disordered nanostructure of CT-CB resulting in lamellae break up, creation of edge sites, and material lost to oxidation, while the longer peripheral lamellae in FCC-CB resist thermal oxidation relative to CT-CB. TEM for both partially oxidized samples supports this interpretation.

Figure 7 shows overview TEM micrographs of the two carbon blacks and Figure 8 shows HRTEM micrographs with arrows pointing towards crumbling particles in Figure 8A,B for CT-CB, while Figure 8C,D show hollowed out intact shells of FCC-CB black.

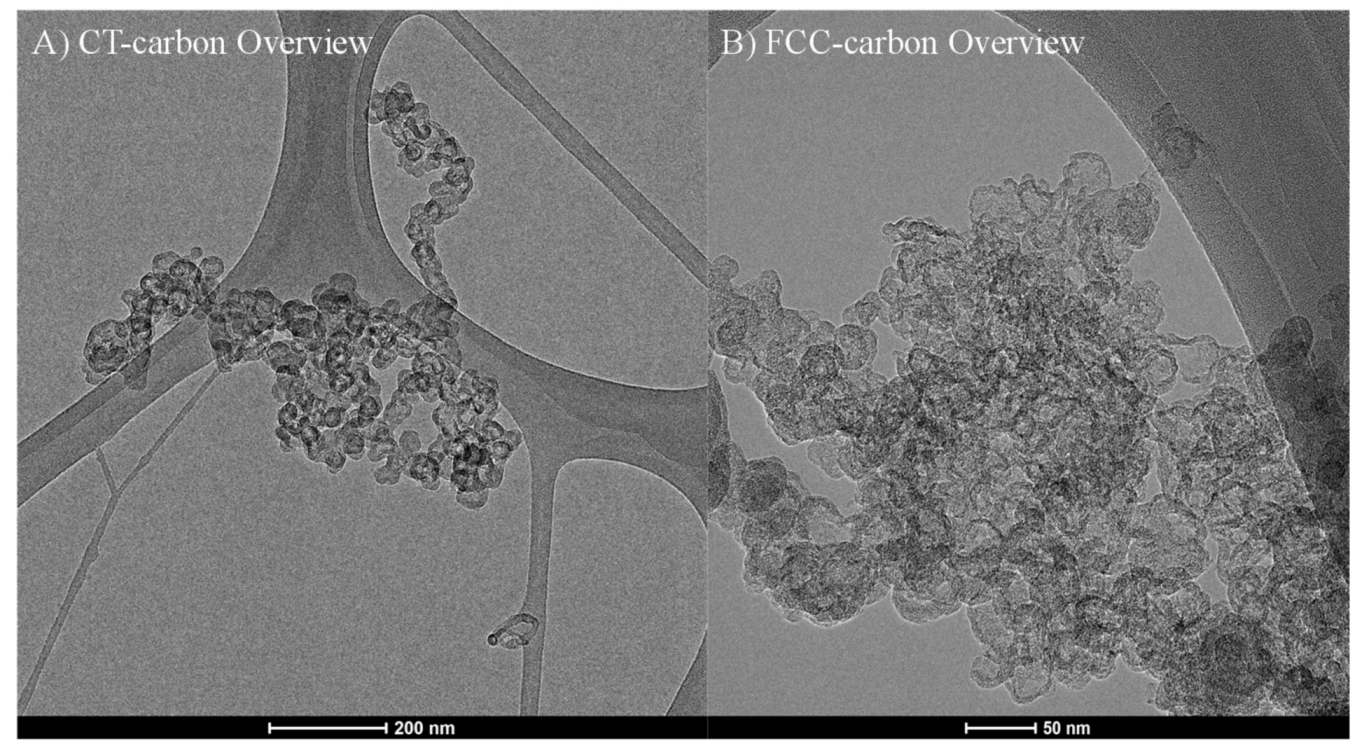

Figure 7. TEM micrographs showing an aggregate of (A) CT- and (B) FCC-CB black after isothermal treatment in a TGA.

The prevalent radial disorder within the particles of CT-CB black results in the lamellae reacting with the available oxygen. The preferential and faster oxidation is consistent with disordered, shorter lamellae comprising the particle perimeter. Such lamellae expose reactive edge sites. Without stable lamellae to support the shell, particle breakup results. On the other hand, FCC-CB shows a hollowed-out interior with a stable shell at the prevalent temperature. The more reactive carbon within the particle's core has preferentially oxidized, while the more stable carbon lamellae at the perimeter have partially annealed. This behavior has previously been observed for carbon blacks isothermally oxidized at temperatures of $\sim 500{ }^{\circ} \mathrm{C}$ [39] and indicates a significant difference in the C-C bonding chemistry between the CT- and FCC-CB blacks. 


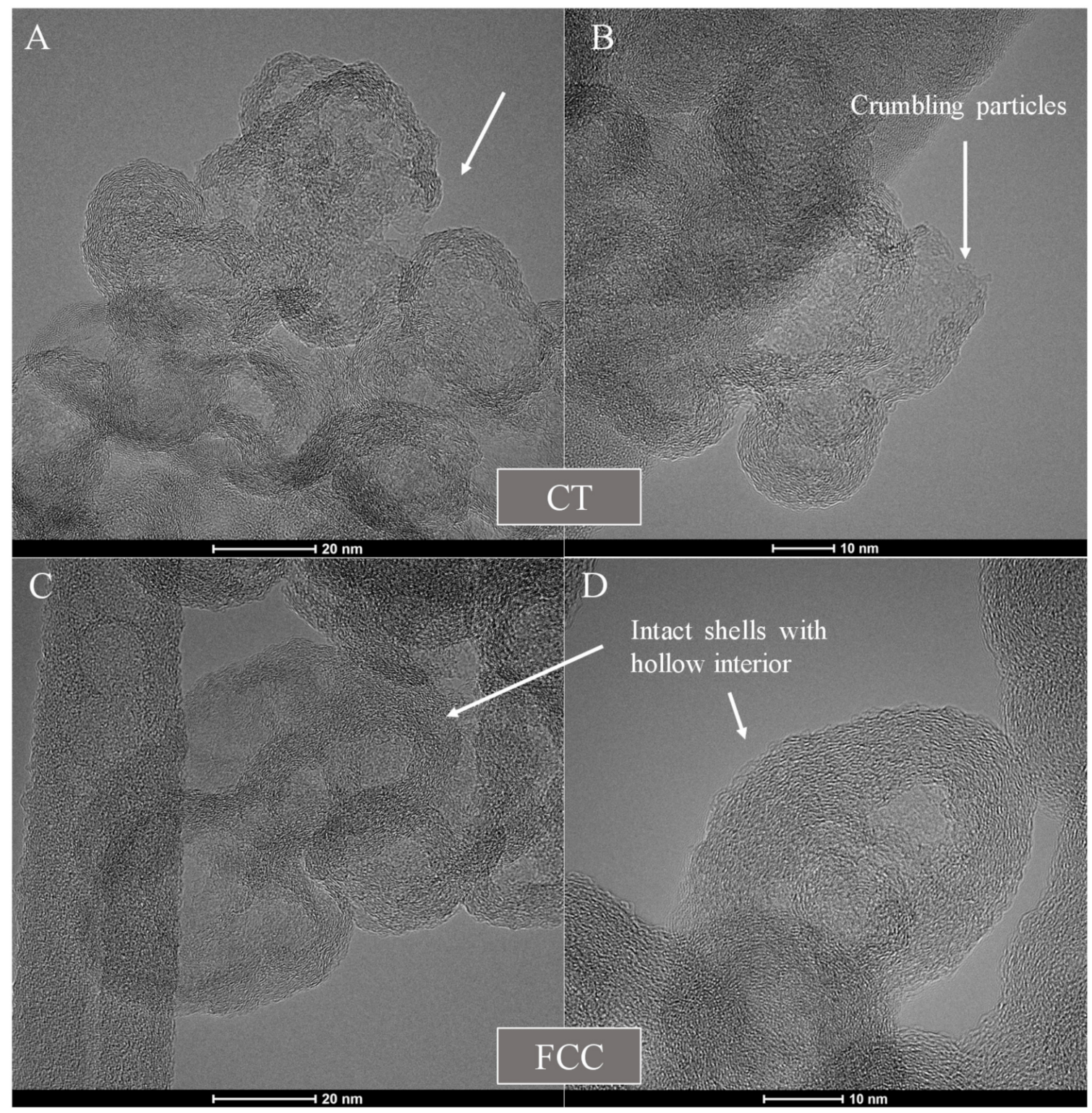

Figure 8. HRTEM micrographs of (A,B) CT- and (C,D) FCC-CB black after isothermal treatment in a TGA.

\subsection{Laser Heat Treatment}

CT- and FCC-CB are laser heated with one $1064 \mathrm{~nm}$ pulse of $170 \mathrm{~mJ} / \mathrm{cm}^{2}$ of the $\mathrm{Nd}$ :YAG laser in an inert $\left(\mathrm{N}_{2}\right)$ atmosphere. Laser heat treatment at extremely short $(\sim 8 \mathrm{~ns})$ timescales followed by microseconds long rapid cooling results in divergent nanostructure for the two carbon blacks. This is premised on source differentiation using laser derivatization [9], where subtle non-obvious differences in the materials' nascent nanostructure and chemistry may be enhanced by laser annealing. Given differences in their nascent material nanostructure, CT- and FCC-CB show a significant divergence in their annealed nanostructures, shown by the following TEM micrographs.

Figure 9 shows micrographs with an overview of the aggregate morphology and structure of the two carbons, followed by Figure 10 showing HRTEM images of the particles after laser annealing. CT-CB shows hollow particles with intact shells, while FCC shows the formation of multiple connected internal ribbons of carbon lamellae.

CT-CB shows a disordered core with a higher $\mathrm{H} / \mathrm{C}$ ratio. Laser heat treatment of this carbon results in a greater extent of carbon vaporization and fragmentation of lamellae within the particle interior. Many smaller lamellae are created in the process, coupled with 
greater mobility that leads to a shell formation as the mobile lamellae move towards the outer perimeter and template of the perimeter shell.

FCC-CB has longer lamellae within a structured particle interior and overall, less radial variation from shell to core. It has a lower core $\mathrm{H} / \mathrm{C}$ ratio and thus, laser heat treatment leads to lesser fragmentation of the longer lamellae. Coupled with lower mobility leads to internal ribbon formation and compartmentalization as seen in Figure 10.

While differences in shell integrity versus crumbling were evident between the FCC-CB and CT-CB materials, respectively, both forms exhibited internal burning during oxidationindicative in each case of more reactive and disordered interior carbon. However, though not substantial, initial, and later stage oxidation rates were consistently different- reflecting differences in interior carbon. In contrast, laser annealing accentuates differences in nanostructure and chemistry yielding starkly different particle structures: a hollow void versus ribbon compartmentalization, as observed in Figure 9. At higher magnification Figure 10 reveals the full differentiation between the two annealed forms. For this reason of differentiation based on differences in formation conditions, the action of the laser may be considered a form of (nano)structural derivatization.

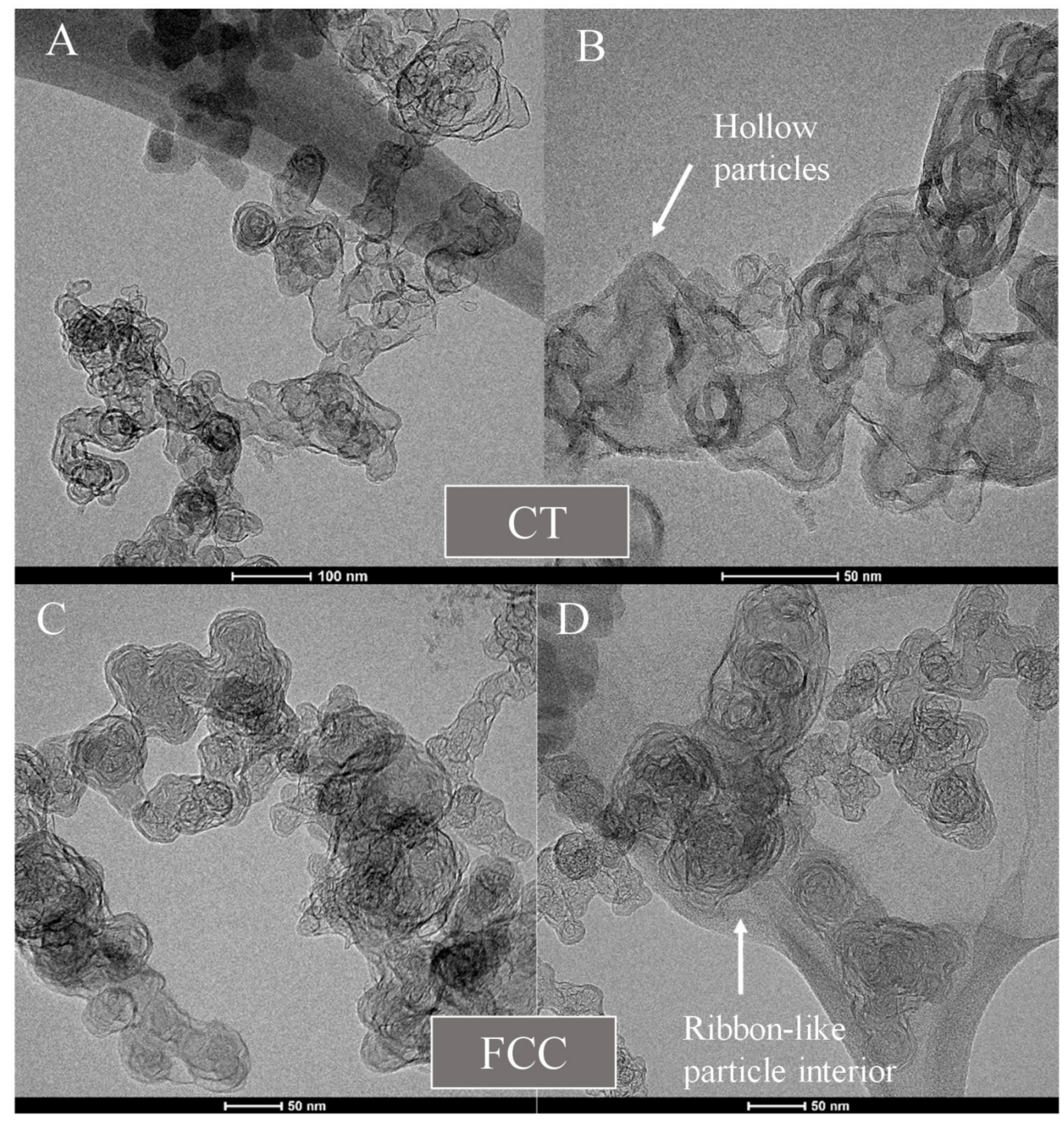

Figure 9. Laser heated aggregates of (A,B) CT- and (C,D) FCC-CB blacks. 


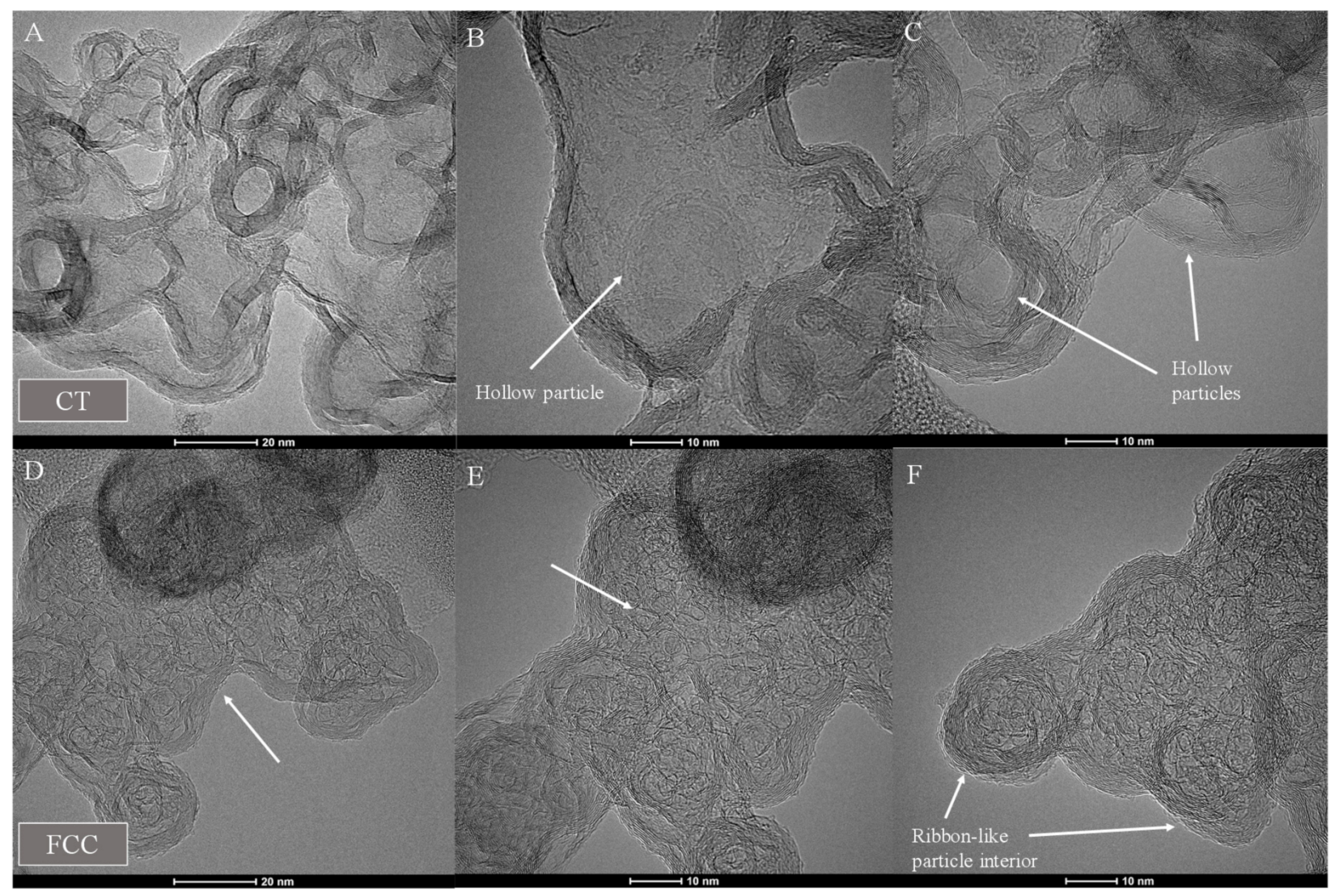

Figure 10. HRTEM images of laser heated (A-C) CT- and (D-F) FCC-CB blacks.

These qualitative inferences are supported by numerical simulations for model compounds. For example, molecular dynamics revealed 1,2,4 Trimethylbenzene (124TMB) to have earlier soot inception and PAH addition reactions than n-propyl benzene, (PBZ) [22]. Consistent with both earlier soot inception and PAH addition, the pyrolysis model for 124TMB predicts earlier pyrene formation, suggesting 124TMB has alternative reaction pathways for pyrene formation. As shown by Chu et al., analysis of the reaction pathway suggested that pyrene is formed via an aromatic radical recombination route, as opposed to the conventional HACA mechanism, a slower route to 2 nd ring formation. These results are consistent with the resonance-stabilized hydrocarbon-radical chain reaction mechanism recently advanced for soot inception and growth $[5,6]$. Radical chain reaction pathways can lead to covalently bound clusters of PAHs and other hydrocarbons that otherwise would be too small to condense at high temperatures, thereby providing a mechanism for rapid particle formation and surface growth [4].

Our observations are consistent with other experimental studies that have shown the formation of incipient particles with carbon-to-hydrogen ratios of $\sim 2$ composed of randomly ordered aromatic structures with some aliphatic character [22]. The resonance stabilized radicals comprising these nuclei react with other hydrocarbon species to form covalently bound complexes that regenerate/maintain resonance-stabilized radical sites [4]. These in turn enable further growth and clustering by continued radical propagation reactions mediated by hydrogen atom abstraction and ejection.

\section{Summary}

Two feedstocks, CT and FCC form carbon black using similar reactor conditions. The main compositional difference is the prevalent content of methylated aromatics, heavy to light in the CT. This difference is postulated to be the driver in early particle inception and growth for the CT feed. With resonance stabilized radicals propagating molecular growth by radical addition reactions, inception begins at earlier times, higher feedstock 
concentrations, and lower temperatures. Collectively these conditions result in rapid nuclei formation, high number concentrations, and early particle growth by nuclei coalescence and $\mathrm{PAH}$ addition. Primary particle cores typically show multiple nuclei.

For the FCC feedstock, higher hydrogen content serves to terminate radicals by $\mathrm{H}-$ abstraction reactions while saturating pyrolysis products (H-atoms and alkyl radicals) can similarly cap aromatic radicals to inhibit their clustering via resonance-stabilized radical (RSR) reactions-both mechanisms delaying particle inception and growth. The lower aromatic content and resulting increased chemical kinetic time allows turbulence induced mixing to lower feed concentration while increasing temperature. With inception occurring under diluted conditions, nuclei concentration is lower and primary particles generally contain but one identifiable center. Smaller pyrolysis species contributing to growth at higher temperatures lead to a more radially uniform nanostructure.

Differences in growth conditions result in different nanostructure and chemistry-as measured by the $\mathrm{sp}^{2}$ content. The comparative $\mathrm{sp}^{2}$ content for the FCC-CB is 3.5 times that of the CT-CB-consistent with early nucleation and growth for the $\mathrm{CT}$ derived particles and delayed nucleation and growth for the FCC derived particles reflecting preceding pyrolysis. Different particle sizes are consistent with this view.

To better illustrate the chemistry and nanostructure differences between the two carbon blacks, pulsed laser annealing is applied. Collectively chemistry, nanostructure, and nuclei differences are magnified. Core-shell structure is observed for the CT-CB while intertwined ribbons comprise the particle interior of the FCC-CB after laser heating. The distinctly different structures support the postulate of different formation conditions for the two carbon blacks. In further support of the postulate and as further illustration of their different chemistry - oxidative reactivity is assessed using a TGA. Disordered carbon reacts faster-with interior burnout observed for the CT-CB material whereas the FCC-CB exhibits mixed surface and internal burnout as observed by TEM.

Author Contributions: Conceptualization, R.L.V.W. and C.R.H.; methodology, R.L.V.W. and C.R.H.; validation, R.L.V.W., J.K. and C.R.H.; formal analysis, M.S. and A.G.; investigation, M.S., A.G., R.L.V.W., J.K. and C.R.H.; resources, R.L.V.W. and C.R.H.; data curation, M.S., A.G., R.L.V.W. and C.R.H.; writing—original draft preparation, M.S., A.G. and R.L.V.W.; writing-review and editing, M.S., A.G., R.L.V.W., J.K. and C.R.H.; visualization, R.L.V.W. and C.R.H.; supervision, R.L.V.W. and C.R.H.; project administration, R.L.V.W. and C.R.H.; funding acquisition, R.L.V.W. and C.R.H. All authors have read and agreed to the published version of the manuscript.

Funding: This work was supported by Birla Carbon, c/o Columbian Chemicals through sponsored agreement number 225973 with the Pennsylvania State University. The APC was funded by MDPI.

Institutional Review Board Statement: Not applicable.

Informed Consent Statement: Not applicable.

Data Availability Statement: Not applicable.

Conflicts of Interest: The authors declare no conflict of interest.

\section{References}

1. D'Anna, A.; Violi, A.; D'Alessio, A.; Sarofim, A.F. A reaction pathway for nanoparticle formation in rich premixed flames. Combust. Flame 2001, 127, 1995-2003. [CrossRef]

2. Siegmann, K.; Sattler, K.; Siegmann, H.C. Clustering at high temperatures: Carbon formation in combustion. J. Electron Spectrosc. Relat. Phenom. 2002, 126, 191-202. [CrossRef]

3. Lai, J.Y.W.; Elvati, P.; Violi, A. Stochastic atomistic simulation of polycyclic aromatic hydrocarbon growth in combustion. Phys. Chem. Chem. Phys. 2014, 16, 7969-7979. [CrossRef] [PubMed]

4. Kholghy, M.R.; Afarin, Y.; Sediako, A.D.; Barba, J.; Lapuerta, M.; Chu, C.; Weingarten, J.; Borshanpour, B.; Chernov, V.; Thomson, M.J. Comparison of multiple diagnostic techniques to study soot formation and morphology in a diffusion flame. Combust. Flame 2017, 176, 567-583. [CrossRef]

5. Vitiello, G.; De Falco, G.; Picca, F.; Commodo, M.; D’Errico, G.; Minutolo, P.; D'Anna, A. Role of radicals in carbon clustering and soot inception: A combined EPR and Raman spectroscopic study. Combust. Flame 2019, 205, 286-294. [CrossRef] 
6. Johansson, K.O.; Head-Gordon, M.P.; Schrader, P.E.; Wilson, K.R.; Michelsen, H.A. Resonance-stabilized hydrocarbon-radical chain reactions may explain soot inception and growth. Science 2018, 361, 997-1000. [CrossRef]

7. Wang, H. Formation of nascent soot and other condensed-phase materials in flames. Proc. Combust. Inst. 2011, 33, 41-67. [CrossRef]

8. Mercier, X.; Carrivain, O.; Irimiea, C.; Faccinetto, A.; Therssen, E. Dimers of polycyclic aromatic hydrocarbons: The missing pieces in the soot formation process. Phys. Chem. Chem. Phys. 2019, 21, 8282-8294. [CrossRef]

9. Singh, M.; Gaddam, C.K.; Abrahamson, J.P.; Wal, R.L.V. Soot differentiation by laser derivatization. Aerosol Sci. Technol. 2018, 53, 207-229. [CrossRef]

10. Betts, W.D. Tar and Pitch. In Kirk-Othmer Encyclopedia of Chemical Technology; John Wiley \& Sons, Inc.: Hoboken, NJ, USA, 2000. [CrossRef]

11. Mochida, I.; Korai, Y.; Hieida, T.; Azuma, A.; Kitajima, E. Detailed Analyses of FCC Decant Oil as a Starting Feedstock for Mesophase Pitch. Fuel Sci. Technol. Int. 1991, 9, 485-504. [CrossRef]

12. Wal, R.L.V.; Tomasek, A.J.; Street, K.; Hull, D.R.; Thompson, W.K. Carbon Nanostructure Examined by Lattice Fringe Analysis of High-Resolution Transmission Electron Microscopy Images. Appl. Spectrosc. 2004, 58, 230-237.

13. Wal, R.L.V.; Tomasek, A.J. Soot oxidation: Dependence upon initial nanostructure. Combust. Flame 2003, 134, 1-9.

14. Wal, R.L.V.; Tomasek, A.J. Soot nanostructure: Dependence upon synthesis conditions. Combust. Flame 2004, 136, 129-140.

15. Wal, R.L.V.; Bryg, V.M.; Hays, M.D. Fingerprinting soot (towards source identification): Physical structure and chemical composition. J. Aerosol Sci. 2010, 41, 108-117.

16. Egerton, R.F. Electron Energy-Loss Spectroscopy in the Electron Microscope; Springer: Berlin/Heidelberg, Germany, 2011.

17. Abrahamson, J.P.; Singh, M.; Mathews, J.P.; Wal, R.L.V. Pulsed laser annealing of carbon black. Carbon N. Y. 2017, 124, 380-390. [CrossRef]

18. Gong, X.; Wang, Z.; Deng, S.; Li, S.; Song, W.; Lin, W. Impact of the Temperature, Pressure, and Particle Size on Tar Composition from Pyrolysis of Three Ranks of Chinese Coals. Energy Fuels 2014, 28, 4942-4948. [CrossRef]

19. Fardhyanti, D.; Damayanti, A. Analysis of Coal Tar Compositions Produced from Sub-Bituminous Kalimantan Coal Tar. World Acad. Sci. Eng. Technol. Int. J. Chem. Mol. Nucl. Mater. Metall. Eng. 2015, 9, 1022-1025.

20. Richards, A.P.; Fletcher, T.H. A comparison of simple global kinetic models for coal devolatilization with the CPD model. Fuel 2016, 185, 171-180. [CrossRef]

21. Schobert, H. Chemistry of Fossil Fuels and Biofuels. Cambridge Series in Chemical Engineering; Cambridge University Press: Cambridge, UK, 2013. [CrossRef]

22. Chu, C.; Zhang, T.; Thomson, M.J. The chemical structure effects of alkylbenzenes on soot formation in a laminar co-flow flame. Combust. Flame 2019, 204, 237-249. [CrossRef]

23. Genetti, D.B. The Chemical Structure Effects of Alkylbenzenes on Soot Formation in a Laminar Co-Flow Flame; Birmingham Young University: Provo, UT, USA, 1999.

24. Filley, R.M.; Eser, S. Analysis of hydrocarbons and sulfur compounds in two FCC decant oils and their carbonization products. Energy Fuels 1997, 11, 623-630. [CrossRef]

25. Tsang, W. The stability of alkyl radicals. J. Am. Chem. Soc. 1985, 107, 2872-2880. [CrossRef]

26. Forrester, A.R.; Hay, J.M.; Thomson, R.H. Organic Chemistry of Stable Free Radicals; Academic Press: London, UK, 1968.

27. Ono, K.; Matsukawa, Y.; Dewa, K.; Watanabe, A.; Takahashi, K.; Saito, Y.; Matsushita, Y.; Aoki, H.; Era, K.; Aoki, T.; et al. Formation mechanisms of soot from high-molecular-weight polycyclic aromatic hydrocarbons. Combust. Flame 2015, 162, 2670-2678. [CrossRef]

28. Mitra, T.; Zhang, T.; Sediako, A.D.; Thomson, M.J. Understanding the formation and growth of polycyclic aromatic hydrocarbons (PAHs) and young soot from n-dodecane in a sooting laminar coflow diffusion flame. Combust. Flame 2019, 202, 33-42. [CrossRef]

29. Pretorius, G.N.; Bunt, J.; Gräbner, M.; Neomagus, H.; Waanders, F.B.; Everson, R.C.; Strydom, C. Evaluation and prediction of slow pyrolysis products derived from coals of different rank. J. Anal. Appl. Pyrolysis 2017, 128, 156-167. [CrossRef]

30. Thomson, M.; Mitra, T. A radical approach to soot formation. Science 2018, 361, 978-979. [CrossRef]

31. Martin, J.W.; Salamanca, M.; Kraft, M. Soot inception: Carbonaceous nanoparticle formation in flames. Prog. Energy Combust. Sci. 2022, 88, 100956. [CrossRef]

32. Frenklach, M.; Mebel, A.M. On the mechanism of soot nucleation. Phys. Chem. Chem. Phys. 2020, 22, 5314-5331. [CrossRef]

33. Rodrigues, P.; Franzelli, B.; Vicquelin, R.; Gicquel, O.; Darabiha, N. Coupling an LES approach and a soot sectional model for the study of sooting turbulent non-premixed flames. Combust. Flame 2018, 190, 477-499. [CrossRef]

34. Bockhorn, H. Soot Formation in Combustion; Springer: Berlin/Heidelberg, Germany, 1994.

35. Baum, T.; Löffler, S.; Löffler, P.; Weilmünster, P.; Homann, K.-H. Fullerene ions and their relation to PAH and soot in low-pressure hydrocarbon flames. Ber. Bunsenges. Phys. Chem. 1992, 96, 841-857. [CrossRef]

36. Kholghy, M.R.; Kelesidis, G.A.; Pratsinis, S.E. Reactive polycyclic aromatic hydrocarbon dimerization drives soot nucleation. Phys. Chem. Chem. Phys. 2018, 20, 10926-10938. [CrossRef]

37. Genetti, D.B. An Advanced Model of Coal Devolatilization Based on Chemical Structure; Birmingham Young University: Provo, UT, USA, 1999. 
38. Yuan, H.; Kong, W.; Liu, F.; Chen, D. Study on soot nucleation and growth from PAHs and some reactive species at flame temperatures by ReaxFF molecular dynamics. Chem. Eng. Sci. 2019, 195, 748-757. [CrossRef]

39. Gaddam, C.K.; Wal, R.L.V.; Chen, X.; Yezerets, A.; Kamasamudram, K. Reconciliation of carbon oxidation rates and activation energies based on changing nanostructure. Carbon N. Y. 2016, 98, 545-556. [CrossRef] 